International Journal of Wireless \& Mobile Networks (IJWMN) Vol. 5, No. 2, April 2013

\title{
A Review on Cooperative Communication Protocols in Wireless World
}

\author{
Juhi Garg ${ }^{1}$, Priyanka Mehta ${ }^{2}$ and Kapil Gupta ${ }^{3}$ \\ ${ }^{1}$ Department of Electronics and Communication Engineering, FET-MITS University, \\ Lakshmangarh, Sikar, India \\ joohigrg@gmail.com \\ ${ }^{2}$ Department of Electronics and Communication Engineering, FET-MITS University, \\ Lakshmangarh, Sikar, India \\ primehta04@gmail.com \\ ${ }^{3}$ Department of Electronics and Communication Engineering, FET-MITS University, \\ Lakshmangarh, Sikar, India \\ kapil_mbmeyahoo.com
}

\begin{abstract}
Future generations of cellular communications requires higher data rates and a more reliable transmission link with the growth of multimedia services, while keeping satisfactory quality of service, . MIMO antenna systems have been considered as an efficient approach to address these demands by offering significant multiplexing and diversity gains over single antenna systems without increasing bandwidth and power. Although MIMO systems can unfold their huge benefit in cellular base stations, but they may face limitations when it comes to their deployment in mobile handsets.

To overcome this drawback, relays (fixed or mobile terminals) can cooperate to improve the overall system performance in cellular networks. Cooperative communications can efficiently combat the severity of fading and shadowing through the assistance of relays. It has been found that using relays the capacity and coverage of cellular networks can be extended without increasing mobile transmit power or demanding extra bandwidth.
\end{abstract}

\section{KEYWORDS}

Cooperative Communication, Coded Cooperation, MIMO, Amplify and Forward, Decode and Forward

\section{INTRODUCTION}

The increasing numbers of users demanding service have encouraged intensive research in wireless communications. The problem with the cooperative communications is the unreliable medium through which the signal has to travel. To mitigate the effects of wireless channel, the idea of diversity has been deployed in many wireless systems [1-3]. Diversity is a communication technique where the transmitted signal travels through various independent paths and thus the probability that all the wireless paths are in fade is made negligible. Frequency diversity, time diversity and space diversity are the three basic techniques for providing diversity to the wireless communication systems.

Multiple-input multiple-output (MIMO) systems, where the transmitters as well as receivers are equipped with multiple antennas, proved to be a breakthrough in wireless communication system which offered new degree of freedom, in spatial domain, to wireless communications. After that, MIMO became part of many modern wireless communications standards like LTE DOI : 10.5121/ijwmn.2013.5209 
International Journal of Wireless \& Mobile Networks (IJWMN) Vol. 5, No. 2, April 2013

Advanced [1], WiMAX [2], [3] and Wireless LAN [4]. However, use of MIMO in small size nodes, like used in wireless cellular networks proved to be a challenge. To address this challenge, idea of cooperative communications came into existence to implement the idea of MIMO in distributed manner. This concept says that transmitting users share each others' antennas to give a virtual MIMO concept. Though, the idea of cooperative communication was given in 2003 by Sendonaris et al. [5], [6], it is still considered an extensive research which is going to exploit its benefits in the next generation communication systems [7][8].

In this article, we discuss a detailed overview of potential benefits offered by this new trend in communication system.

\section{WIRELESS COMMUNICATIONS AND MIMO SYSTEMS}

Wireless communication systems have grown successfully over the last few decades and are expected to continue in the future. There is an increasing demand for high data rates today in order to support high speed interactive internet services and advanced multimedia applications such as mobile TV, online gaming etc. This trend is enormously evolving in the $4 \mathrm{G}$ systems.

However, transmission over wireless channel of high rate (i.e. bandwidth) faces fundamental limitations due to impairments inflicted by wireless channel such as, path loss, shadowing and fading effects. These impairments can be compensated by various ways such as by increasing transmit power, bandwidth, and/or applying powerful error control coding (ECC). However, power and bandwidth are very scarce and expensive radio resources whereas ECC yields reduced transmission rate. Thus, acquiring a high data rate together with reliable transmission over error-prone wireless channels is a major challenge for wireless system designer.

Another way to cope with the impairments offered by the wireless channel is the use of MIMO systems. In MIMO systems, multiple antennas are used at the transceiver. This arrangement can significantly increase data rate and reliability of the wireless link. MIMO systems use either VBLAST (Vertical Bell Laboratories Layered Space-Time) or DBLAST (Diagonal Bell Labs Layered Space-Time) algorithm. However, using multiple co-located antennas causes degradation in the system Quality of service (QoS) due to correlation between them. Also, due to size, cost, or hardware limitations, small handheld wireless devices may not be able to support multiple antennas [10].

To overcome the above drawback, an innovative approach known as cooperative communication has been suggested to exploit MIMO's benefit in a distributed manner. Such a technique is also called a virtual MIMO, since it allows single antenna mobile terminals to reap some of the benefits of MIMO systems. This concept is illustrated in Fig-1 below [9][10].

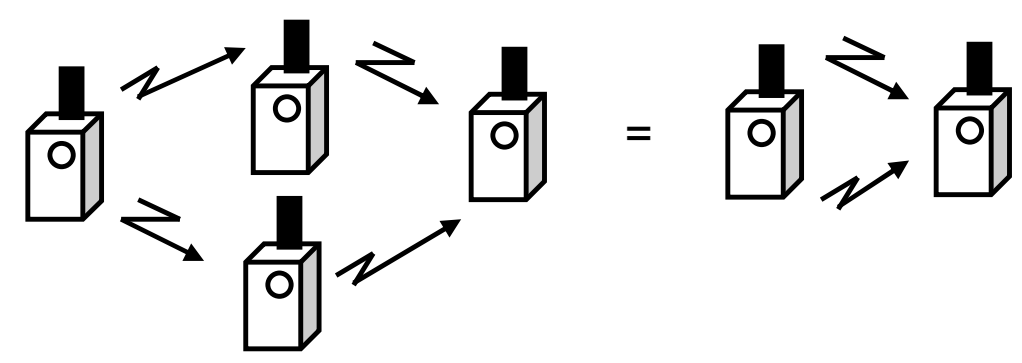

Figure 1. Illustration of MIMO and virtual MIMO systems [10] 


\section{WORKING PRINCIPLE}

The idea of cooperation was presented by van der Meulen in 1971, which established foundation of relay channel. Cooperative communication takes advantage of broadcast nature of the wireless medium where the neighbouring nodes overhear the source's signals and relay the information to the destination. Thus, idea of creating a VAA came into existence [11].

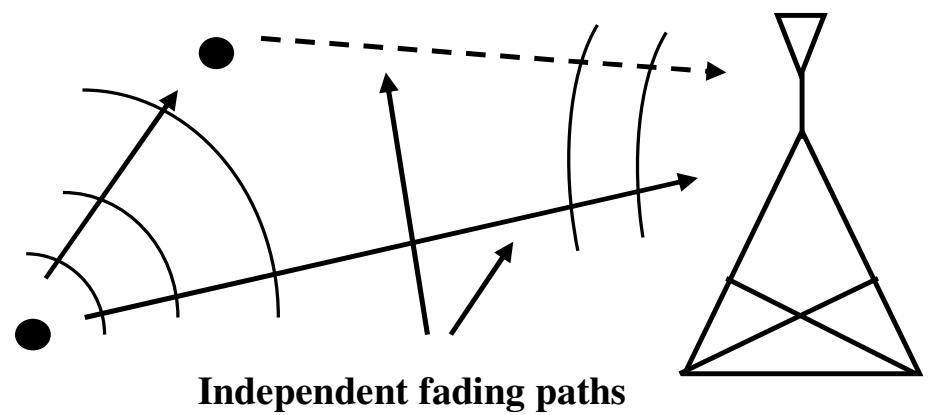

Figure 2. Cooperative Communication

\section{HISTORICAL BACKGROUND}

The scheme of cooperative communications is based on simple three terminal relay channel introduced by Van der Meulen [12][13]. In this, it was shown that both the transmitter and receiver nodes can be aided by a relay node to improve the rate region of the transmitter. Later, idea of cooperative communications was given by Sendonaris [5][6]. The authors prove that cooperative communications improves the achievable rate for both users, based on two user's cooperative communication scenario.

This is further extended by Lane man et al. [14-16]. The authors introduced the idea of amplifyand-forward, decode-and-forward, and dynamic decode-and-forward protocol. The outage probability is used as a performance metric to advocate the idea of cooperative communication.

The concept of coded cooperation was given by Hunter [17], [18] which is given as the integration of cooperative communication and channel coding. The users code their information into block composed of two parts. The transmitting user transmits the first part of block and second part is transmitted by another cooperating user if it receives the first part successfully, otherwise, user itself transmits the second part of the block. In this way, diversity gain is exploited to efficiently counter the wireless channel impairment.

Later, various channel coding schemes have been applied to this scenario. LDPC [19] was first applied by Khojastepour et al. [20]. Turbo codes et al. [21] was investigated by various researchers [22-24]. Extensive work has been implemented by Hunter et al. [17], [18] where they have utilized convolution codes to cooperative communication.

Thus, in the wireless transmission, the transmission of a node is physically broadcasted. Hence, even though a node is not the next-hop node of a transmitted data, it can overhear it. In the conventional transmission, only the next-hop node of the received data accepts it and all other nodes just ignore it. We call this conventional transmission a direct transmission in this paper. 


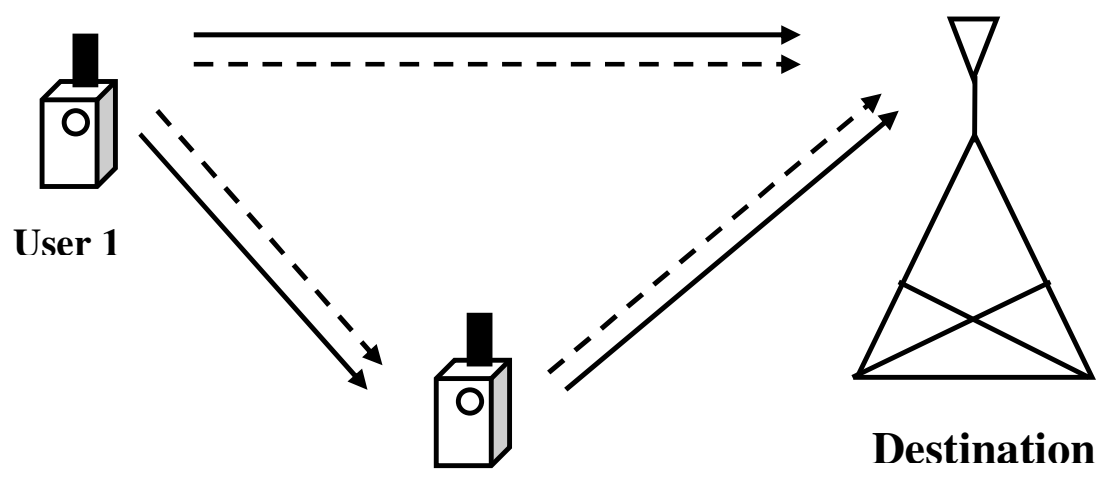

User 2

Figure 3. In Cooperative Communication each mobile is both a relay and a user

However, if we appropriately utilize the broadcasting nature of the wireless transmission, we can improve the performance of transmission such as its reliability and throughput. The cooperative transmission uses this broadcasting nature of wireless transmission to improve the performance of the transmission. In the cooperative transmission, if a node overhears a data transmission, it may forward it to its next-hop node after performing some processing that depends on cooperative transmission scheme. We call this node a cooperative node. Hence, the next-hop node of the data may receive multiple data from the original transmitter node and from several cooperative nodes and by appropriately combining and decoding them, the performance of the transmission could be improved compared with that of the direct transmission.

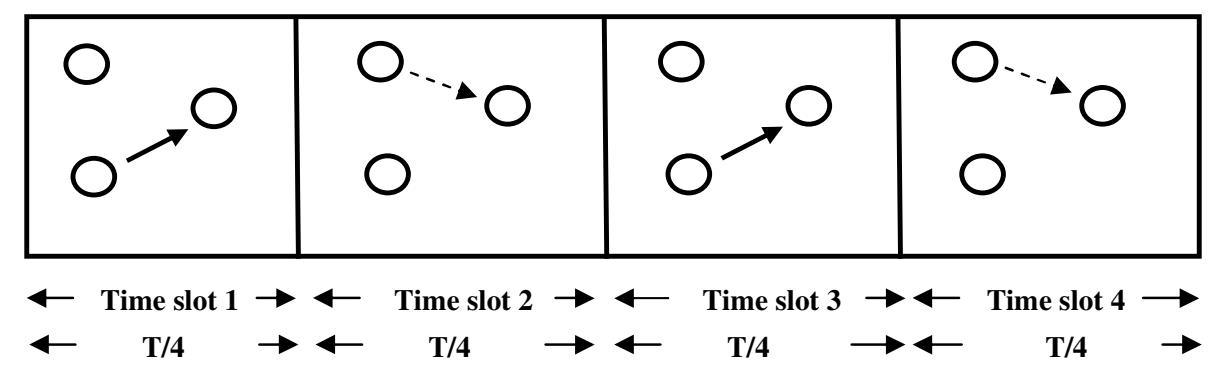

(a) Direct transmission in a frame

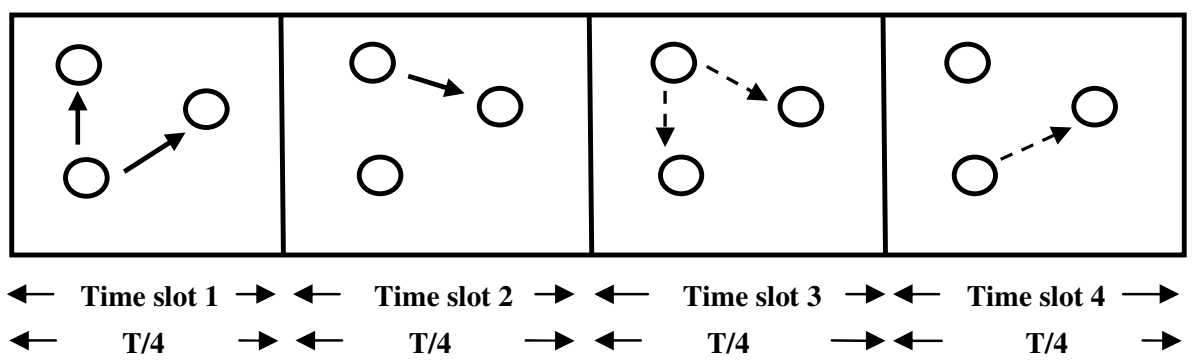

(b) Cooperative transmission in a frame

Figure 4. Direct and Cooperative Communication in a frame 


\section{PREVIOUS WORK}

Cooperative relaying is an effective technique to combat multipath fading and enhance system capacity and coverage. This section provides a literature survey on various aspects of cooperative relaying.

Initially, capacity analyses of Gaussian relay channel was comprehensively studied in [25] . In [26] , various relaying strategies for wireless networks have been studied. End-to-end error and outage performance of non-regenerative relay network have been studied in [27] . Here, all nodes are equipped with single antenna which operates in Nakagami-m fading channel. Outage probability of multi-hop amplifies and forward relay systems have been analyzed in [28] . In [29], closed form expressions of average bit error rate (ABER), amount of fading and outage probability (OP) have been derived for Rayleigh-fading channel where relay works in nonregenerative mode and destination performs equal gain combining (EGC). In [30] and [31], closed-form expressions are derived for the moment generating function (MGF), probability density function (PDF), and cumulative distribution function (CDF) of the statistically independent Gamma random variables. An amplify-and-forward (AF) relay network has been studied in [32] where a source communicates with the user having best channel conditions through an intermediate relay that serves to multiple users. Spatial reuse of relay time-slot in interference limited channel has been analyzed in [33] for AF cooperative relaying. In [34] , Laneman et al. has developed and analyzed distributed space-time coded cooperative diversity protocols for improving spectral efficiency. Error performance has been analyzed in [35] when cooperative relay system operates in asymmetric channels. In [36], outage performance of MIMO relay channel has been investigated when source and relay use same orthogonal space time block code. Selection cooperation in network scenario has been discussed in [37] where multiple sources transmit their message and support each other to forward signals to their respective destinations. End-to end error performance of selection cooperation has been presented in [38] . In [39], optimum power allocation for multi-antenna relay network at the expense of increased computational complexity has been presented.

An overview of various cooperation schemes related to their implementation has been discussed in [40] . In [41], closed form expressions of OP and bit error rate (BER) for binary phase shift keying (BPSK) are derived for case where communication between source and destination is supported by multiple-antenna relay and both relay and destination performs MRC of signals in Rayleigh fading channel. OP and average error rate of two-hop multi-antenna relay based system for the case when relay performs selection combining (SC) of signals and destination performs MRC of signals are analyzed in [42] and [43], respectively. In [44], closed form expressions for OP and BER have been derived when multi-antenna relay network operates in correlated Nakagami-m fading channel and both the relay as well as destination performs MRC of signal. Closed form expression of outage probability has been derived in [45]; here, communication between source and destination is supported by two multi-antenna relay nodes. A new efficient scheme for cooperative wireless networking based on linear network codes has been discussed in [46] .

\section{BASIC RELAYING PROTOCOLS}

In cooperative communications, the transmitting user not only broadcast their own message but they also relay information, on behalf of each other, to the destination. The strategy, by which the information is relayed to the destination, is known as protocol. Various protocols have been introduced so far. Here, we describe some of the basic relaying protocols. 


\subsection{Amplify and Forward}

This is the simplest protocol. Here, the information received by a user from original transmitter is amplified and then forwarded to the destination. Based on the principle of amplifying repeaters [47], amplify-and-forward protocol was formally introduced by Lane man et al. [48]

\subsection{Decode and Forward}

In this relaying protocol the partner users decodes the message received from original transmitter, re-encodes and then forwards it to the destination. Thomas M. Cover and Abbas A. El Gamal are considered as its pioneer [50] and later the idea was further explored by many authors with the name of Decode-and-Forward [16], [15], [50].

\subsection{Compress and Forward}

In this relaying protocol, the message is decoded from the transmitter and the partner user forwards a compressed version of it to the destination, so as to get the diversity benefits [49].

\section{COOPERATIVE RELAYING TECHNIQUES}

In this section, we will discuss Different Cooperative Protocols or Transmissions Techniques used in Cooperative Communication. Cooperative communications protocols can be generally categorized into fixed relaying schemes and adaptive relaying schemes. In this section, we describe both of these schemes along with both single relay and multi relay scenario.

\subsection{Cooperation Protocols}

A cooperation strategy is modelled into two orthogonal phases, to avoid interference between the two phases, either in TDMA or FDMA.

In phase 1, source broadcast information to its destination, and the information is also received by the relay (due to broadcast) at the same time as shown in figure 5 below.

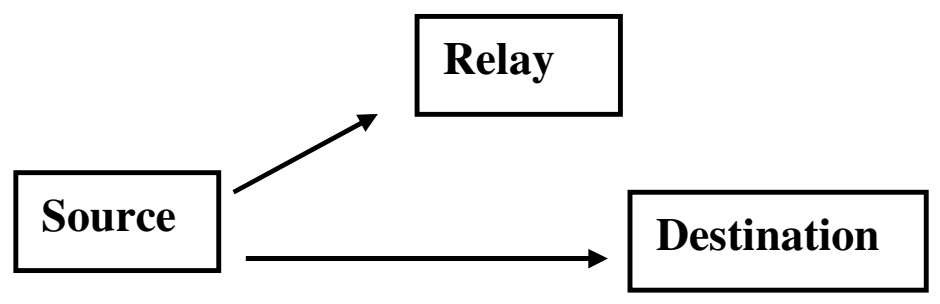

Figure 5. Phase 1

In phase 2, the relay forwards or retransmits the information to the destination as shown in figure 6 below.

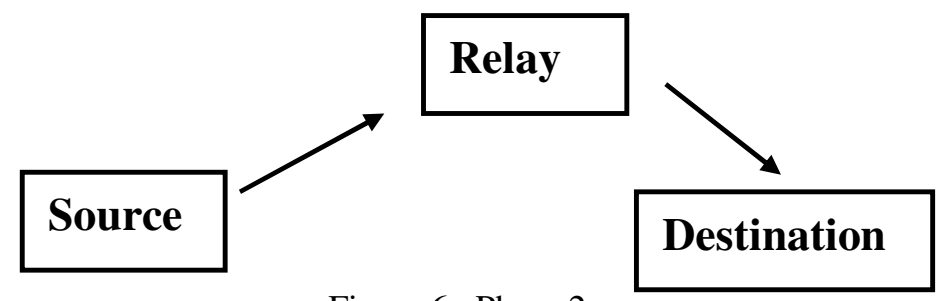

Figure 6. Phase 2 
Fig- 7 below depicts a general relay channel, where the source transmits with power P1 and the relay transmits with power P2. Here, we will consider the special case when both source and relay transmit with equal power P. Optimal power allocation is a vast topic so can be consider for future work.

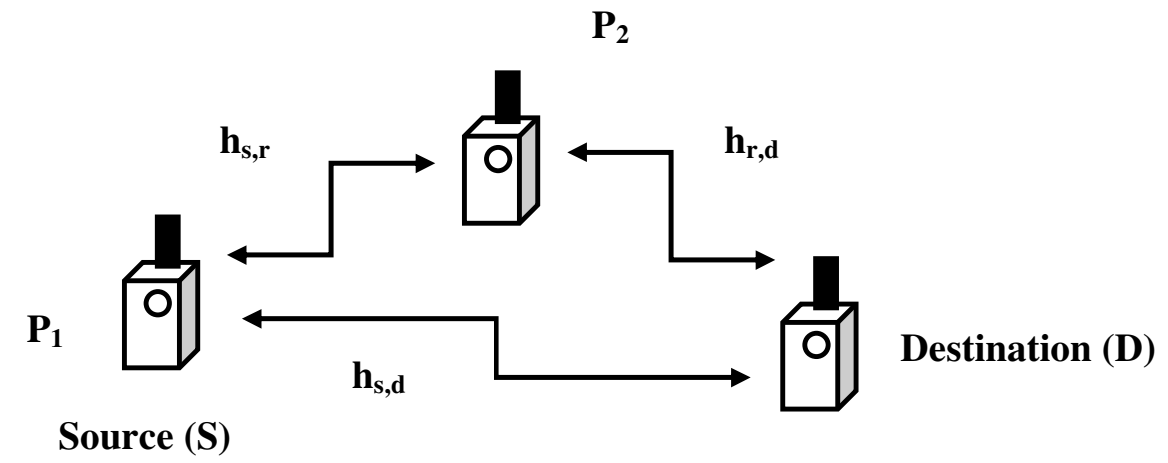

Figure 7. Simplified Cooperation Model [51]

In phase 1, source forwards its information to both the destination and the relay. The received signals $Y_{s d}$ at the destination and $Y_{s r}$ at the relay, can be written as

$$
\begin{aligned}
& Y_{s d}=\sqrt{P} h_{s d} x+n_{s d} \\
& Y_{s r}=\sqrt{P} h_{s r} x+n_{s r}
\end{aligned}
$$

where $\mathrm{P}$ is the transmitted power at source, $\mathrm{x}$ is the transmitted information symbol, and $n_{s d}$ and $n_{s r}$ are additive noise. In (1) and (2), $h_{s d}$ and $h_{s r}$ are the channel fades between the source and destination and the relay, respectively, and are modelled as Rayleigh flat fading channels. Rayleigh flat fading channel can be mathematically modelled as complex Gaussian random variable. It is given as $\mathrm{z}=\mathrm{x}+\mathrm{jy}$, where real and imaginary parts are zero mean independent and identically distributed (i.i.d) Gaussian random variables. The noise terms $n_{s d}$ and $n_{s r}$ are modelled as zero-mean complex Gaussian random variables with variance No.

In phase 2, relay forwards source's signal to the destination, and this can be modelled as

$$
Y_{r d}=h_{r d} q\left(Y_{s r}\right)+n_{r d}
$$

where the function $\mathrm{q}(\cdot)$ depends upon processing which is implemented at the relay node [52].

\subsection{Fixed Cooperation Strategies}

In fixed relaying, channel resources are divided between source and relay in a fixed (deterministic) manner. The processing at relay differs according to the employed protocols. The most common techniques are the fixed AF relaying protocol and the fixed relaying DF protocol [15][34]. 


\subsubsection{Fixed Amplify \& Forward (Single Relay)}

In a fixed AF relaying protocol, or AF protocol, the relay scales the received version and transmits its amplified version to the destination. The amplify-and-forward scheme is presented in Fig-8.

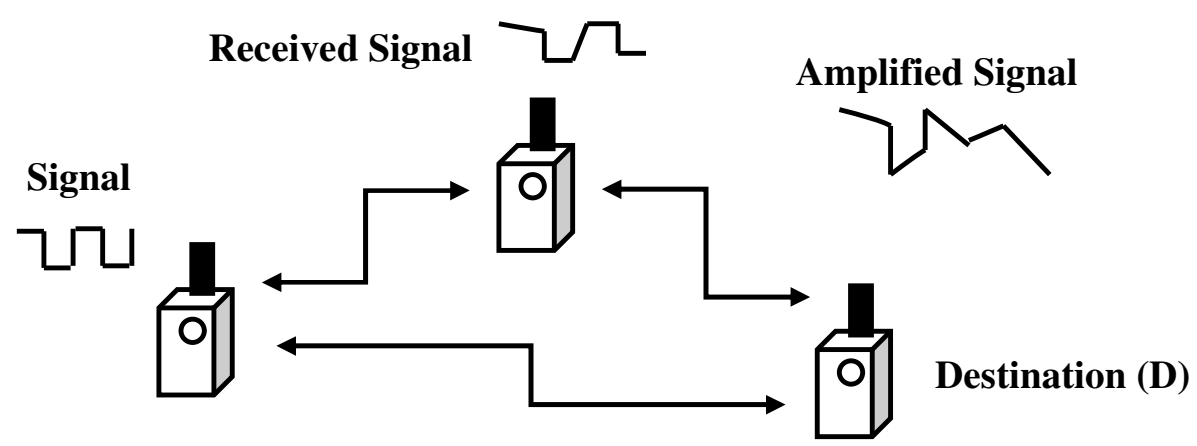

Figure 8. Amplify and Forward System Model [51]

The amplify-and-forward relay channel can be modelled as follows. The signal transmitted from the source $\mathrm{x}$ to both the relay and destination can be given as

$$
Y_{s r}=\sqrt{P} h_{s r} x+n_{s r} \text { and } Y_{s d}=\sqrt{P} h_{s d} x+n_{s d}
$$

where $h_{s r}$ and $h_{s d}$ are channel fades between the source and the relay and destination, respectively. The terms $n_{s r}$ and $n_{s d}$ denote the additive white Gaussian noise with zero-mean and variance $\mathrm{N}_{0}$. Here, the relay amplifies the source signal and forwards it to the destination, to equalize the effect of the channel fades between the source and the relay. The relay scales the received signal by a factor that is inversely proportional to the received power, which is denoted by

$$
\beta=\frac{\sqrt{P}}{\sqrt{P\left|h_{s r}\right|+N_{o}}}
$$

The signal transmitted from relay is therefore, given by $\beta Y_{s r}$ and has power $\mathrm{P}$ equal to the power of the signal transmitted from the source. In phase 2, the relay amplifies the received signal from source and forwards it to the destination.

The received signal, in phase 2 , at the destination, according to (4) is given as

$$
Y_{r d}=\frac{\sqrt{P}}{\sqrt{P\left|h_{s r}\right|+N_{o}}} h_{r d} Y_{s r}+n_{r d}
$$

Here $h_{r d}$ is the channel coefficient from relay to the destination and $n_{s d}$ is an additive noise. 


\subsubsection{Fixed Amplify \& Forward (Multi Relay)}

An amplify-and-forward protocol does not suffer from any error propagation problem because the relay does not need to perform any hard-decision operation on the received signal. First we discuss the multi-node amplify-and-forward protocol and then analyze its two relaying strategies. In the first phase, each relay forwards the source's signal to the destination, while in the second phase each relay forwards a combined signal from source and previous relays. Fig- 9 shows the multi-node Amplify and Forward system model.

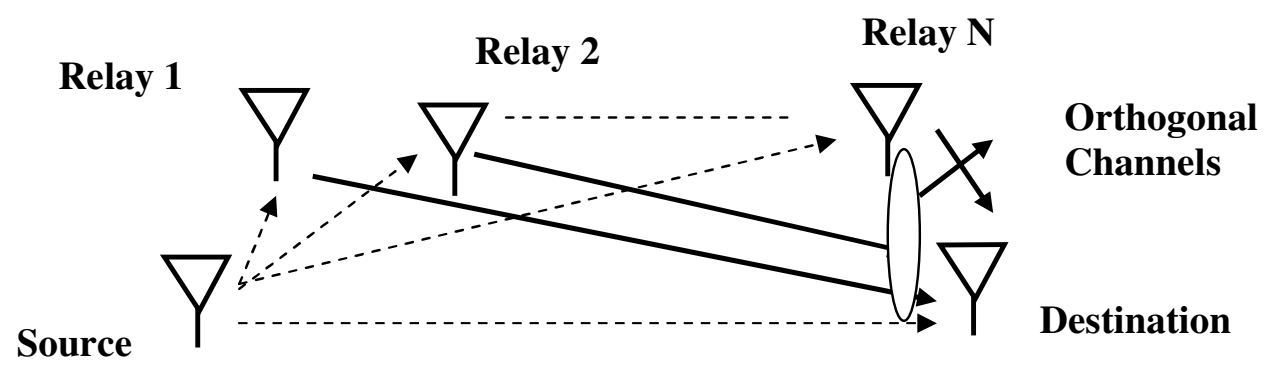

Figure 9. Multi Node Amplify and Forward System Model [52]

\subsubsection{Fixed Decode and Forward (Single Relay)}

Another possibility at the relay node is to decode the received signal at the relay, re-encode it, and then retransmit it to the receiver. The decode-and-forward scheme is presented in Fig-10. This kind of relaying is often called as DF scheme. If the decoded signal at the relay is denoted by x', the transmitted signal from the relay can be denoted by x', given that x' has unit variance.

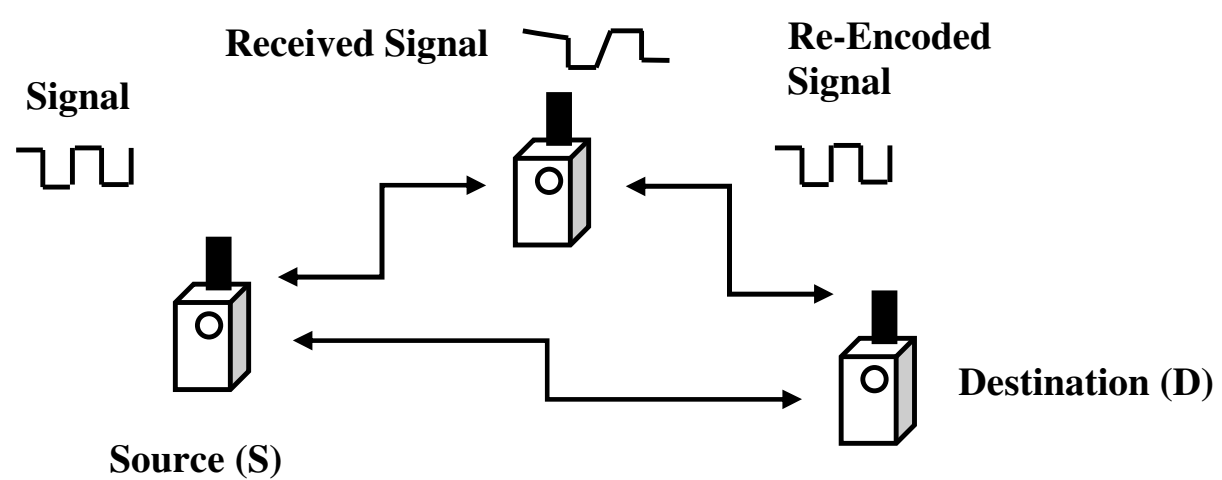

Figure 10. Decode and Forward System Model [52]

If an incorrect signal is forwarded to the destination, then decoding at the destination is meaningless. It is clear that the diversity achieved for such a scheme is only one, because performance of the system is limited by the worst link from the source-relay and sourcedestination. 
International Journal of Wireless \& Mobile Networks (IJWMN) Vol. 5, No. 2, April 2013

Although fixed DF relaying has the advantage of reducing the effects of additive noise at the relay over AF relaying, it forwards erroneously detected signals to the destination, causing error propagation that can diminish the performance of the system. Mutual information between the source and destination is limited by the mutual information of the weakest link between the source-relay and the combined channel from the source-destination and relay-destination. The received signal at the destination in Phase 2 can be given as

$Y_{r d}=\sqrt{\beta_{2}} h_{r d} x+n_{r d}$

With the help of channel coefficients $h_{s d}$ (between source and destination) and $h_{r d}$ (between relay and destination), the destination detects the transmitted symbols by jointly combining the received signal $Y_{s d}$ from the source in eq. (1) and $Y_{r d}$ from the relay in eq. (7).

\subsubsection{Fixed Decode and Forward (Multi Relay)}

Here, we consider a N-relay (2 Relay For Simulation Purpose) wireless network, where information is to be transmitted from a source to a destination. Due to the broadcast nature of the wireless channel, relays overhear the transmitted information and thus cooperate with the source to send its data. The wireless link between any two nodes is modelled as a Rayleigh fading channel with AWGN.

The channel fades for different links are assumed to be statistically independent because the relays are usually spatially separated. The additive noise at receiving terminals is modelled as zero-mean, complex Gaussian random variables with variance $\mathrm{N}_{\mathrm{o}}$. The relays are assumed to transmit over orthogonal channels, thus there is no inter-relay interference.

In DF protocol cooperation strategy, each relay can combine the signal received from the source along with the signals transmitted by previous relays, decode it and then retransmit it to the receiver after re-encoding it again. A general cooperation scenario, denoted as $\mathrm{C}(\mathrm{m})(1 \leq \mathrm{m} \leq \mathrm{N}$ $-1)$, is implemented in which each relay combines the signals received from $\mathrm{m}$ previous relays along with that received from the source.

The multi relay decode and forward scenario is shown in Fig- 11, in which each relay combines the signals received from previous relays along with that received from the source. In all considered cooperation scenarios, destination coherently combines the signals received from the source and relays. Now, we are presenting the system model for a general cooperative scheme $\mathrm{C}(\mathrm{m})$ for any $1 \leq \mathrm{m} \leq \mathrm{N}-1$, where each relay decodes the information after combining the signals received from the source and previous $m$ relays. We consider 2 relays for simulation purpose. 


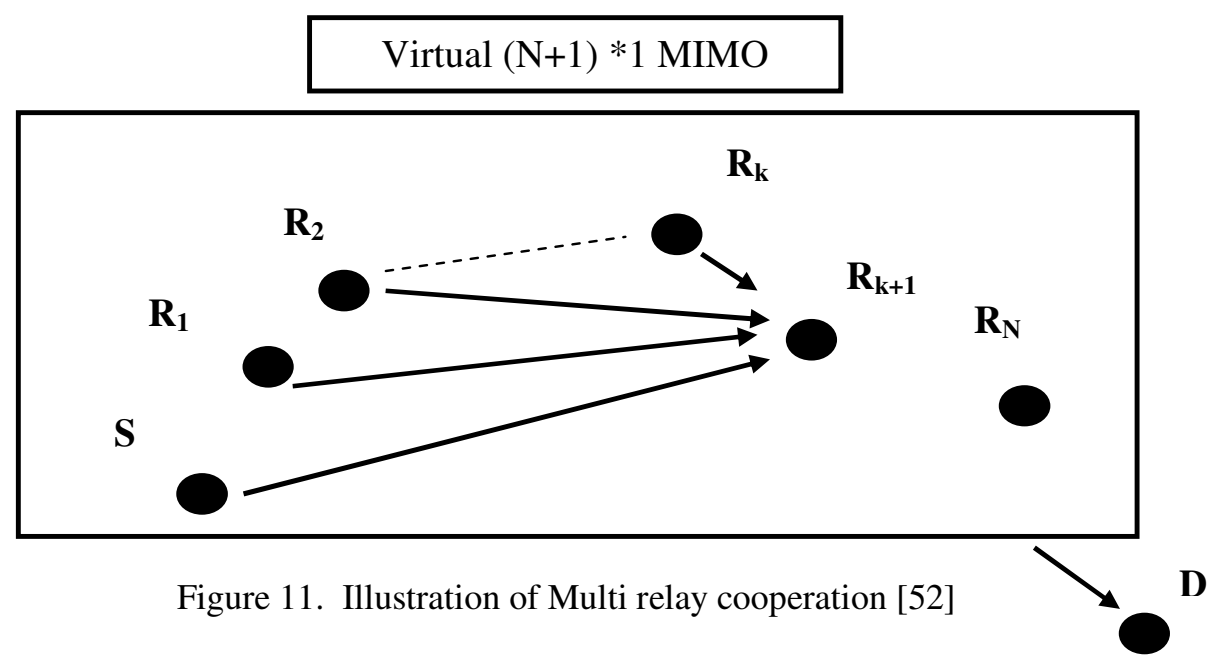

In General Multi Node cooperation protocol has $(\mathrm{N}+1)$ phases as stated in AF section but three in our case. In phase 1 , source transmits information to both destination and the $\mathrm{i}^{\text {th }}(1 \mathrm{st})$ relay, which can be modelled as,

$$
\begin{aligned}
& Y_{s d}=\sqrt{P_{o}} h_{s d} x+n_{s d} \\
& Y_{s, r i}=\sqrt{P_{o}} h_{s r i} x+n_{s r i} \quad 1 \leq i \leq N
\end{aligned}
$$

where $P_{o}$ is the power transmitted at the source, $\mathrm{x}$ is the transmitted symbol, $h_{s d}$ and $h_{s r i}$ are the fading coefficients between source and destination, and $\mathrm{i}^{\text {th }}$ relay, respectively. The terms $n_{s d}$ and $n_{s r i}$ denote the AWGN channel noise. In phase 2, the 1st relay decodes the signal it receives from source, re-encode $\&$ send it to other relay $\&$ the destination. Second relay combines the received signals from the 1st relay and source as follow

$$
Y_{r 2}=\sqrt{P_{o}} h_{s, r 2}^{*} Y_{s, r 2}+\sqrt{P_{i}} h_{r 1, r 2}^{*} Y_{r 1, r 2}
$$

where $h_{r 1, r 2}$ is the channel fading coefficient between the 1st and the 2nd relay, $Y_{r 1, r 2}$ denotes the signal received at 2 nd relay from the 1 st relay, which is given as

$$
Y_{r 1, r 2}=\sqrt{P_{i}} h_{r 1, r 2} x+n_{r 1, r 2}
$$

where $P_{i}$ is the power transmitted at relay 1 , finally in phase $(\mathrm{N}+1)$, the destination coherently combines all of the received signals, which are modelled as

$Y_{d}=\sqrt{P_{o}} h_{s, d}^{*} Y_{s d}+\sum_{i=1}^{N} \sqrt{P_{i}} h_{r i, d}^{*} Y_{r i, d}$ 
International Journal of Wireless \& Mobile Networks (IJWMN) Vol. 5, No. 2, April 2013

\subsection{Other Cooperation Strategies}

Besides the techniques for fixed relaying, there are other techniques, such as compress-and forward cooperation and coded cooperation. Here, we discuss an overview of each of them.

\subsubsection{Compress and Forward}

The main difference between compress-and forward and decode/amplify-and-forward is that in the later the relay transmits a copy of the received message, while in compress and forward the relay transmits a quantized and compressed version of the received message. Therefore, the destination performs the reception function by combining the received message from both source node and its compressed version from the relay node.

The quantization and compression process at relay node is a process of source encoding, i.e., representation of each received message as a sequence of symbols. Let us assume that these symbols are binary digits (bits). At the destination, an estimate of the compressed message is obtained by decoding the received sequence of bits. This decoding operation involves mapping of received bits into a set of values that estimate the transmitted message. This mapping process normally involves the introduction of distortion, which can be considered as a form of attenuation and noise [25], [53].

\subsubsection{Coded Cooperation}

Coded cooperation differs from the previous schemes such that the cooperation is implemented at the level of the channel coding subsystem. We know in both amplify and forward and decode-and-forward schemes, the relay repeats the bits sent by the source. In coded cooperation incremental redundancy at relay, which when combined at the receiver with the codeword sent by the source, results in a codeword with larger redundancy [54].

\subsection{Adaptive Cooperation Strategies}

Fixed relaying has advantage of easy implementation and disadvantage of low bandwidth efficiency. This is because half of the channel resources are allocated to relay for transmission, which leads to reduced overall rate, because in such a scenario, a high percentage of the packets transmitted by the source to the destination could be received correctly by the destination and the relay's transmissions would be wasted.

To overcome this problem, adaptive relaying protocols are developed to improve the inefficiency. Here, we will discuss two strategies: selective DF relaying and incremental relaying [52].

\subsubsection{Selective DF Relaying}

In this relaying scheme, the relay decodes the received signal and forwards decoded information to the destination, if the signal-to noise ratio of a signal at the relay exceeds a certain threshold. On the other hand, if the channel between the source and the relay suffers, such that the signalto-noise ratio falls below the threshold, the relay remains idle. Selective relaying shows improvement upon the performance of fixed DF relaying, as the threshold at the relay can be used to overcome the inherent problem in fixed DF relaying in which the relay forwards all decoded signals to the destination although some signals are incorrect. We knew, if the SNR in source-relay link exceeds the threshold, the relay is able to decode source's signal correctly. In this case, SNR of the combined MRC signal at destination is given as sum of the received SNR from the source and the relay. 
International Journal of Wireless \& Mobile Networks (IJWMN) Vol. 5, No. 2, April 2013

\subsubsection{Incremental Relaying}

In incremental relaying, there is a feedback channel from the destination to the relay. The destination sends an acknowledgement to the relay if it was able to receive the source's message correctly. This protocol has the best spectral efficiency among the previously described protocols because the relay does not always need to transmit, and hence second transmission phase becomes opportunistic depending on the channel state condition of channel between the source and the destination. Nevertheless, incremental relaying achieves a diversity order of two as mentioned below:

In incremental relaying, if the source transmission in the first phase was successful, then there is no second phase and source transmits new information in the next time slot. On the other hand, if source transmission was not successful in the first phase, then relay can use any of the fixed relaying protocols to transmit the source signal from the first phase. The transmission rate (R) is random in incremental relaying. If the first phase was successful, the transmission rate is $\mathrm{R}$, while if it was in outage, the transmission rate becomes $\mathrm{R} / 2$ as in fixed relaying.

\section{APPLICATIONS}

This section highlights some of the areas where the cooperative relaying strategies can be applied.

\subsection{Virtual antenna array}

The field of high-data-rate, spectrally efficient and reliable wireless communication, is currently receiving much attention. It is a well known fact that the use of MIMO antenna system improves the diversity gain of wireless systems. However, multi-antenna technique is not attractive for tiny wireless nodes due to limited hardware and signal processing capability. Diversity can be achieved through user cooperation, whereby mobile users share their physical resources to create a virtual array, which thus, removes the burden of multiple antennas on wireless terminals.

\subsection{Wireless ad-hoc network}

Ad hoc network is a self organizing network without any centralized infrastructure. In this $n / w$, distributed nodes form a temporary functional network and support seamless leaving or joining of nodes. Such network has been deployed for military communication and civilian applications including commercial and educational use etc.

\subsection{Wireless sensor network}

Lifetime of sensor network can be increased by deploying cooperative relaying hence, energy consumption in sensor nodes got reduced. We knew, communication through weaker channels requires huge energy as compared to relatively strong channels. So, careful incorporation of cooperating relay nodes into routing process can select better communication links and precious battery power can be saved.

\subsection{Cooperative sensing for cognitive radio}

In cognitive radio system, secondary users can utilize the resources which are employed for licensed primary users. When primary users want to use their licensed resources, secondary users have to vacant these resources. Thus, secondary users have to constantly sense the presence of primary user. Probability of false alarming can be reduced with the help of spatially distributed nodes, which thus improve the channel sensing reliability by sharing the information [55]. 
International Journal of Wireless \& Mobile Networks (IJWMN) Vol. 5, No. 2, April 2013

\section{TRACKS FOR FUTURE WORK}

This paper investigated the performance of some of the basic cooperative relaying schemes. For practical implementation of such schemes in a network environment, it is necessary to investigate several other issues, some of which are briefly outlined below:

\subsection{System modelling taking inference into consideration}

In a network scenario, cooperative relay system suffers from co-channel and adjacent channel interference. Thus, the effect of the interference needs to be analysed.

\subsection{Power optimization based on link condition}

Wireless nodes generally have limited battery power. Power can be allocated if relay based systems have some feedback mechanism, based on link condition [34]. Such dynamic allocation may save battery power or boost the data transfer rate and hence the optimization area to be investigated.

\subsection{Full duplex operation of relays}

Relay operating in half-duplex mode creates a wide system bandwidth expansion. Full-duplex relay operating in single frequency are used to solve this problem. Thus, the effect of fullduplex relay operation needs to be investigated.

\subsection{Complexity performance trade off}

Relays can process the signal in non-regenerative or regenerative mode depending on their functionality. Non-regenerative mode puts less processing burden on the relay as compared to regenerative mode of operation; hence, it is often preferred when complexity and/ or latency are needed to be analysed. Scope has been found to exist in future for non-regenerative mode of relay operation. Noise amplification is a major issue in this operation.

\subsection{Modelling of relay link in various environments}

Wireless propagation suffers with path loss, shadowing and fading which depends on regional geography. Statistical behaviours of various propagation environments are available in literatures. Relay link can be easily modelled in such propagation environment.

\subsection{Relay selection}

In the present state of art, wireless relay nodes are half-duplex in nature. Participation of large number of relay increase the diversity order, but spectrum efficiency of system may suffer. Therefore, selection of the nodes and optimum number of nodes which should be selected to optimize the performance in terms of rate and reliability is an interesting field of research.

\subsection{Spectrum efficiency}

Orthogonal transmission from relay to receiver in TDMA mode can affect the spectrum efficiency. Relay can interact with the receiver in CDMA mode which avoids bandwidth expansion. Therefore, orthogonal code design for distributed relay node may be possible area for research.

\subsection{Node cooperation with cross-Layer design}

Node cooperation is an efficient technique to improve the performance of WSN's. Sensor nodes are powered by small battery that cannot be easily recharged. So reducing energy consumption 
International Journal of Wireless \& Mobile Networks (IJWMN) Vol. 5, No. 2, April 2013

is a wide issue for such type of network. Protocol layering provides modularity that facilitates standardization and implementation. Unfortunately, layering precludes the benefits of joint optimization across protocol layers; hence, precious battery power cannot be efficiently utilized. In this regard, it would be interesting to investigate cross-layer optimization [56] .

\subsection{Bidirectional user cooperation}

In this work, we are assuming that relay terminals do not have their own data and they are just forwarding data received from source. In user cooperation, user's terminal not only transmits its own data, but also relays other user's data by sharing some of the resources. In this regard, it would be interesting to investigate bidirectional user cooperation extension.

\subsection{Base station cooperation}

In single cellular system, user terminals can communicate with parent base station. Users near the outskirts of cell can communicate with neighbouring base stations and thus becomes a source for generating interfering signals. To overcome this, neighbouring base stations can also cooperate with parent base station and perform joint decoding of received signals. The work presented for relay cooperation can be extended for base station cooperation.

\section{CHALlengeS}

Helping out users in a cooperative fashion has its price. Here, we will describe the challenges that incur in systems with cooperative communication incorporated [57][58].

\subsection{Complex Schedulers}

Relaying requires more sophisticated schedulers since not only traffic of different users and applications needs to be scheduled but also the relayed data flows. This function decides how many resources are scheduled for a single user (or relay node). This function affects the achieved throughput of the system. Practical implementations of a scheduler also consider Automatic Repeat-Request (ARQ) protocols and Quality-of-Service (QOS) classes, which experience different priorities in the scheduling process.

\subsection{Increased Overhead}

A full system functioning requires handovers, synchronization, extra security, etc. This clearly induces an increased overhead w.r.t to a system that does not use relaying.

\subsection{Increased Interference}

If the offered power savings are not used to decrease the transmission power of the relay nodes but rather to boost capacity or coverage, then relaying will certainly generate extra intra and inter-cell interference, which potentially causes the system performance to deteriorate. So cooperative relying is more suitable for $3 \mathrm{G} / 4 \mathrm{G}$ systems which are more tolerant to interference. Many Interference mitigation schemes have been proposed for cooperative communication e.g., successive interference cancellation (SIC) scheme in wireless communication network. Zero forcing (ZF) or minimum mean square error (MMSE) receivers are used to mitigate co-channel interference $(\mathrm{CCI})$ in the interference cancellation strategy. For efficient utilization of scarce radio spectrum and codes, a centralized medium access control (MAC) protocol is proposed to coordinate the code assignment and channel access among the users and relays [59], [60]. 
International Journal of Wireless \& Mobile Networks (IJWMN) Vol. 5, No. 2, April 2013

\subsection{Increased End-To-End Latency}

Relaying typically involves the reception and decoding of the entire data packet before it can be retransmitted. If delay-sensitive services are being supported, such as voice or the increasingly popular multimedia web services, then the latency induced by the decoding may become detrimental. Latency increases with the number of relays and also with the use of interleavers, such as utilized in GSM voice traffic. To circumvent this latency, either simple transparent relaying (i.e. AF relaying) or some advanced decoding methods need to be used.

\subsection{More Channel Estimates}

The use of relays effectively increases the number of wireless channels. This requires the estimation of more channel coefficients and hence more pilot symbols need to be provided if coherent modulation was to be used.

\section{CONCLUSIONS}

As stated at the outset, the field of high-data-rate, efficient and reliable wireless communication, is currently receiving much attention. Cooperative transmission is emerging as an effective technique for combating effects of path loss, shadowing, and multi-path fading. This tutorial elaborates wireless cooperative communication, a technique that both allow single antenna mobiles to share their antennas and reap the benefits of multiple antenna systems. Cooperative relaying provides diversity gain, reduces outage probability and improves BER performance. Various types of relays, mode of operation, applications, and tracks for future work have been discussed here. This paper will be helpful for incorporating relay based system in real scenario. Throughout this paper we focused on only two Protocols viz AF \& DF but there are many other protocols as well that deserve attention. We also didn't include Power Allocation i.e. at what power; source/relay should transmit without causing interference for others.

\section{REFERENCES}

[1] 3GPP TR 36.814 V1.2.1, "Further advancements for EUTRA: Physical layer aspects," Technical Specification Group Radio Access n/w, June 2009.

[2] I. P802.16j/D9, "Draft amendment to IEEE standard for local and metropolitan area network part 16: Air interface for fixed and mobile broadband wireless access systems: Multihop relay specification," May 2009.

[3] Yang Yang; Honglin Hu; Jing Xu; Guoqiang Mao, "Relay technologies for WiMAX and LTEAdvanced mobile systems," IEEE Comm. Magazine, vol. 47, no. 10, pp. 100-105, October 2009.

[4] "IEEE draft standard for local and metropolitan area networks; part 11: Wireless LAN medium access control (MAC) and physical layer (PHY) specification: amendment: enhancements for higher through- put, IEEE Draft Std.802.11n (d2)," 2007.

[5] A. Sendonaris, E. Erkip and B. Aazhang, "User cooperation diversity- Part I: System description," IEEE Transaction on Communications, vol. 51, no. 11, pp. 1927-1938, Nov. 2003.

[6] A. Sendonaris, E. Erkip and B. Aazhang, "User cooperation diversity-Part II: Implementation aspects and performance analysis," IEEE Trans. on Commun., vol. 51, no. 11, pp. 1939-1948, Nov. 2003.

[7] L. Sankar, G. Kramer and N. B. Mandayam, "Dedicated-relay vs. user cooperation in timeduplexed multi-access networks," Journal of Communications, vol. 6, no. 9, pp. 330-339, July 2011.

[8] Hongzheng Wang; Shaodan Ma; T. S. Ng, "On performance of cooperative communication systems with spatial random relays," IEEE Trans. on Commun., vol. 59, no. 4, pp. 1190-1199, 2011. 
International Journal of Wireless \& Mobile Networks (IJWMN) Vol. 5, No. 2, April 2013

[9] Birsen, Sirkeci, "Distributed Distributed Cooperative Communication in Large-Scale Wireless Networks," Cornell University 2006.

[10] Quang Trung Duong, “On Cooperative Communications and Its Application to Mobile Multimedia,” Blekinge Institute of Technology, ISBN: 978-91-7295-167-9, 2010.

[11] Y.W. Peter Hong, Wan-Jen Huang \& C.C. Jay Kuo, "Cooperative communications and Networking: Technologies and System Design,” Springer, (2010) ISBN: 1441971939.

[12] E. C. Van Der Meulen, "Transmission of information in a T-terminal discrete memory less channel,” Ph.D. Disser., Dept. of Statistics, University of California, Berkeley, 1968.

[13] Edward C. Van Der Meulen, "Three terminal communication channels," Adv. Appl. Prob., vol. 3, pp. 120-154, 1971.

[14] J. N. Laneman and G. W. Worn ell, "Energy-efficient antenna sharing and relaying for wireless networks," in IEEE Wireless Commun. and Networking Conference (WCNC-2000), vol. 1, pp. 7-12, Sep 2000.

[15] J. N. Laneman, D. N. C. Tse and G. W. Wornell, "Cooperative diversity in wireless networks: efficient protocols and outage behaviour," IEEE Trans. on Information Theory, vol. 50, no. 12, pp. 3062-3080, Dec. 2004.

[16] J. N. Laneman, "Cooperative diversity in wireless networks: Algorithms and architectures," Ph.D. dissertation, Massachusetts Institute of Technology, Cambridge, MA, Aug 2002.

[17] T. E. Hunter and A. Nosratinia, "Co-operation diversity through coding," IEEE International Symposium on Information Theory, pp. 220, July 2002.

[18] T. E. Hunter and A. Nosratinia, "Diversity through coded co-operation," IEEE Transactions on Wireless Commun., vol. 5, no. 2, pp. 283-289, Feb. 2006.

[19] R. G. Gallager, "Low density parity-check code," Ph.D. Dissertation, Massachusetts Institute of Technology, Cambridge, MA, 1963.

[20] M. A. Khojastepour, N. Ahmed and B. Aazhang, "Code design for the relay channel and factor graph decoding," Conference Record of the Thirty-Eighth Asilomar Conf. On Signals, Systems and Computers, vol. 2, pp. 2000-2004, 2004.

[21] C. Berrou, A. Glavieux and P. Thitimajshima, "Near Shannon limit error-correcting coding and decoding: Turbo-codes," IEEE International Conference on Communications, vol. 2, pp. 10641070, 1993.

[22] Z. Zhang and T. M. Duman, "Capacity-approaching turbo coding and iterative decoding for relay channels," IEEE Trans. on Commun., vol. 53, no. 11, pp. 1895-1905, Nov. 2005.

[23] B. Zhao and M. C. Valenti, "Distributed turbo-coded diversity for the relay channel," IEEE Electronics Letters, vol. 39, no. 10, pp. 786-787, May 2003.

[24] Bin Zhao and Matthew C. Valenti, "Cooperative diversity using distributed turbo codes," Virginia Technical Symposium on Wireless Personal Communications, (Blacksburg, VA), June 2003.

[25] T. Cover and A. E. Gamal, "Capacity theorems for the relay channel," IEEE Trans. on Information Theory, vol. 25, no. 5, pp. 572-84, Sep. 1979.

[26] P. Herhold, E. Zimmer-mann and G. Fettweis, "Cooperative multi-hop transmission in wireless networks," Computer N/w, Vol. 49, no. 3, pp. 299-324, 2005. Selected Paper from the European Wireless 2004 Conference.

[27] M. S. Alouini and M. O. Hasna, "End-to-end performance of transmission systems with relays over Rayleigh-fading channels", IEEE Trans. on Wireless Commun., vol. 2, no. 6, pp. 1126-31, Nov. 2003.

[28] C. Conne and Il-Min Kim, "Outage probability of multi-hop amplify-and-forward relay systems," IEEE Trans. on Wireless Communications, vol. 9, no. 3, pp. 1139-49, Mar. 2010. 
International Journal of Wireless \& Mobile Networks (IJWMN) Vol. 5, No. 2, April 2013

[29] S. Ikki and M. H. Ahmed, "Performance analysis of cooperative diversity using equal gain combining (EGC) technique over Rayleigh fading channels," IEEE International Conf. on Commun., pp. 5336-41, Jun. 2007.

[30] G. K. Karagiannidis, T. A. Tsiftsis, R. K. Mallik, N. C. Sagias and S. A. Kotsopoulos, "Closedform bounds for multi-hop relayed communications in Nakagami-m fading", IEEE International Conf. on Communications, vol. 4, pp. 2362-6, May 2005.

[31] G. K. Karagiannidis, T. A. Tsiftsis and R. K. Mallik, "Bounds for multi-hop relayed communications in Nakagami-m fading," IEEE Trans. on Communications, vol. 54, no.1, pp. 18-22, Jan. 2006.

[32] J. B. Kim and D. Kim, "Comparison of two SNR-based feedback schemes in multi-user dualhop amplify and forward relaying networks", IEEE Commun. Letters, vol. 12, no.8, pp. 557- 9, Aug. 2008.

[33] A. Agustin and J. Vidal, "Amplify-and-forward cooperation under interference-limited spatial reuse of the relay slot", IEEE Trans. on Wireless Commun., vol. 7, no. 5, pp. 1952-62, May 2008.

[34] J. N. Laneman and G. W. Wornell, "Distributed space-time-coded protocols for exploiting cooperative diversity in wireless networks", IEEE Trans. on Information Theory, vol. 49, no. 10, pp. 2415-25, Oct. 2003.

[35] A. Adinoyi and H. Yanikomeroglu, "On the performance of co-operative wireless fixed relay in asymmetric channels,” IEEE Global Telecommun. Conf., pp. 1-5, Dec. 2006.

[36] L. Vandendorpe and B. K. Chalise, "Outage probability analysis of a MIMO relay channel with orthogonal space-time block codes," IEEE Commun. Letters, vol. 12, no. 4, pp. 280-2, Apr. 2008.

[37] E. Beres and R. Adve, "Selection cooperation in multi-source co-operative networks", IEEE Trans. on Wireless Communications, vol. 7, no. 1, pp. 118-27, Jan. 2008.

[38] A. Adinoyi, Y. Fan, H. Yanikomeroglu and H. V. Poor, "On the performance of selection relaying,” IEEE Vehicular Technology Conference, pp. 1-5, Sep. 2008.

[39] A. Talebi and A. W. A. Krzymien, "Optimized power allocation for multiple-antenna multi-relay cooperative communication system," IEEE International Conf. on Spread Spectrum Techniques and Applications, pp. 222-226, Aug. 2008.

[40] H. Katiyar, R. Bhattacharjee and B. S. Paul, "User cooperation in TDMA wireless system," IETE Technical Review Journal, vol. 25, pp. 270-6, Sep. 2008.

[41] H. Katiyar and R. Bhattacharjee, "Performance of MRC combining multi-antenna co-operative relay network," AEU - International Journal of Electronics and Communications (Elsevier), vol. 64, no. 10, pp. 988-91, 2010.

[42] R. Bhattacharjee and H. Katiyar, "Outage performance of two-hop multi-antenna co-operative relaying in Rayleigh fading channel," IEEE Electronics Letters, vol. 45, no.17, pp. 881-3, Aug. 2009.

[43] H. Katiyar and R. Bhattacharjee, "Average error rate of multi-antenna decode and forward cooperative relay network,” IEEE INDICON, DAIICT Ahmadabad, pp. 18-20, 2009.

[44] R. Bhattacharjee and H. Katiyar, "Performance of two-hop regenerative relay network under correlated Nakagami-m fading at multi-antenna relay," IEEE Commun. Letters, vol. 13, no. 11, pp. 820-2, Nov. 2009.

[45] H. Katiyar and R. Bhattacharjee, "Outage performance of multi-antenna relay co-operation in the absence of direct link,” IEEE Commun. Letters, vol. 15, no. 4, pp. 398-400, Apr. 2011.

[46] M. Xiao and M. Skoglund, "Multiple-user cooperative communications based on linear network coding," IEEE Trans. on Commun., vol. 58, no. 12, pp. 3345-51, Dec 2010.

[47] B. Schein and R. Gallager, "The Gaussian parallel relay network," pp. 22, June 2000. 
International Journal of Wireless \& Mobile Networks (IJWMN) Vol. 5, No. 2, April 2013

[48] J. N. Laneman, G. W. Wornell, and D. N. C. Tse, "An efficient protocol for realizing cooperative diversity in wireless networks," IEEE Intern. Symposium Information Theory, pp. 294, June 2001.

[49] A. E. Gamal and T. Cover, "Capacity theorems for the relay channel," IEEE Trans. on Information Theory, vol. IT-25, no. 5, pp. 572-584, Sep. 1979.

[50] G. D. Menghwar and C. F. Mecklenbräuker, "Cooperative versus non-cooperative communications," in 2nd International Conference on Computer, Control and Communication, 2009 (IC4 2009), Karachi, Pakistan, Feb. 2009.

[51] K. Alexopoulos, "Performance Analysis of Decode-and-Forward with Cooperative Diversity and Alamouti Cooperative Space-Time Coding in Clustered Multihop Wireless Networks," N.P.S Monterey California, 2008.

[52] K. J. Rayliu, A. K. Sadek, Weifengsu, and Andres Kwasinski, "Cooperative Communications and Networking,” Cambridge University Press, ISBN-13 978-0-511-46548-2, 2009.

[53] G. Kramer, M. Gastpar and P. Gupta, "Co-operative strategies and capacity theorems for relay networks,” IEEE Trans. on Information Theory, 51(9):3037-306, pp. 3037-3063, Sept. 2005.

[54] M. Janani, A. Hedayat, T. E. Hunter and A. Nosratinia, "Coded cooperation in wireless communications: space-time transmission and iterative decoding," IEEE Trans. on Signal Processing, 52:362-370, February 2004. pp. 362-371.

[55] E. Peh and Y. C. Liang, "Optimization for co-operative sensing in cognitive radio networks", IEEE Wireless Commun. and Networking Conference, pp. 27-32, Mar. 2007.

[56] Y. Q. Zhang, “Cross-layer design for QoS support in multi-hop wireless networks”, IEEE Trans. on Signal Processing, vol. 96, no. 1, pp. 64-76, Jan. 2008.

[57] A. Nosratinia, T. E. Hunter and A. Hedayat, "Cooperative Communication in Wireless Networks," IEEE Commun. Magazine, vol. 42, no. 10, pp. 74-80, 2004.

[58] Mischa Dohler, Yonghui Li, “Cooperative Communications Hardware, Channel \& Phy," John Wiley \& Sons, 2010 ISBN 978-0-470-99768-0

[59] Eun Cheol Kim and Jae Sang Cha, "Successive interference cancellation for cooperative communication systems," International Conference on Hybrid Information Technology, 2009, pp. 652-656.

[60] Kuang-Hao Liu, Hsiang-Yi Shin and Hsiao-Hwa Chen, "Interference-resistant cooperative wireless networks based on complementary codes,” John Wiley \& Sons, 2009.

\section{Authors}

Juhi Garg was born in India on July 12, 1989. She received her B. Tech. Degree in Electronics and Communication Engineering from A.C.E.I.T., Jaipur (University RTU, KOTA), Rajasthan, India in 2011 and currently is a M. Tech. (Signal Processing) student in Mody Institute of Technology and Science (MITS, Deemed University), Rajasthan, India.

Priyanka Mehta was born in India on December 4, 1989. She received her B. Tech. Degree in Electronics and Communication Engineering from Marudhar Engineering College, Bikaner (University RTU), Rajasthan, India in 2010 and currently is a M. Tech. (Signal Processing) student in Mody Institute of Technology and Science (MITS, Deemed University), Rajasthan, India.

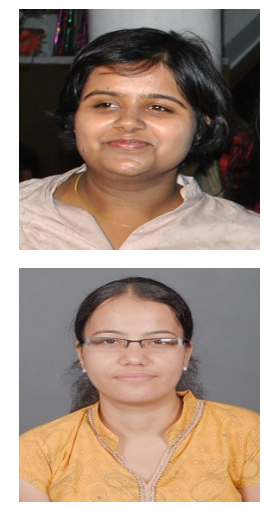


International Journal of Wireless \& Mobile Networks (IJWMN) Vol. 5, No. 2, April 2013

Kapil Gupta was born in Kota (Rajasthan), India in 1980. He received his B.E. (Hons.) degree in Electronics \& Communication Engineering in 2003 from RU and M.E. (Hons.) degree in Digital Communication from JNV University, Jodhpur, Rajasthan in 2008. He is an Assistant Professor of Mody Institute of Technology and Science (Deemed University), Lakshmangarh and has 7 years of experience in teaching. His research interests are in Digital Communication over fading channel and Error correction codes.

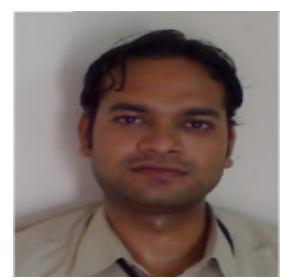

